\title{
Uncertainty in soft constraint problems
}

\author{
Student: Maria Silvia Pini \\ Supervisor: Francesca Rossi \\ University of Padova, Italy. E-mail: \{mpini\}@math.unipd.it
}

Preferences and uncertainty occur in many real-life problems. We are concerned with the coexistence of preference and uncertainty in the same problem. In particular, we consider uncertainty, defined by the theory of possibility [2], that is one non-probabilistic way of dealing with uncertainty, that comes from lack of data or imprecise knowledge.

We propose a method to integrate fuzzy preferences and uncertainty [3], which follows the approach in [2] and allows one to handle uncertainty within a fuzzy optimization engine, which at the same time observing separately the preference and the robustness of complete instantiations. To order the complete instantiations, we define three semantics, which correspond to the attitude to the risk.

We generalize this method in [4] to deal also with other classes of preferences such as probabilistic or weighted constraints [1], and also probabilistic uncertainty. In particular, probabilistic constraints are very useful for modelling real-life scenarios where fuzzy constraints are not the ideal setting, because combining preferences multiplying them is better than taking their minimum value. Weighted constraints are useful to model problems where preferences are penalties (or costs) to be added, and where the best preferences are the smallest ones.

We plan to develop a solver that can handle problems with several classes of soft constraints, together with uncertainty expressed via possibility or probability distributions. The solver will be able to generate solution orderings according the our semantics as well as others that we will define by following different optimistic or pessimistic approaches.

We also plan to develop techniques for reasoning with uncertainty in problems with both positive and negative preferences.

Acknowledgements. This is a joint work with K. Brent Venable (University of Padova, Italy).

\section{References}

1. S. Bistarelli, U. Montanari and F. Rossi. Semiring-based Constraint Solving and Optimization. Journal of the ACM, Vol.44, n.2, March 1997.

2. D. Dubois, H. Fargier, H. Prade. Possibility theory in constraint satisfaction problems: handling priority, preference and uncertainty. Applied Intelligence, 6,1996.

3. M.S. Pini, F. Rossi, B. Venable. Possibility theory for reasoning about uncertain soft constraints. Proc. ECSQARU 2005, Springer LNAI 3571 , 2005.

4. M.S. Pini, F. Rossi, B. Venable. Possibilistic and probabilistic uncertainty in soft constraints problems. Proc. of the IJCAI 2005 Multidisciplinary Workshop on Advances in Preference Handling, Edinburgh, July 2005. 